\title{
Metamaterials: transforming theory into reality
}

\author{
Natalia M. Litchinitser ${ }^{1, *}$ and Vladimir M. Shalaev ${ }^{2}$ \\ ${ }^{1}$ Department of Electrical Engineering, The State University of New York at Buffalo, Buffalo, New York 14260, USA \\ ${ }^{2}$ School of Electrical and Computer Engineering and Birck Nanotechnology Center, Purdue University, \\ West Lafayette, Indiana 47907, USA \\ *Corresponding author: natashal@buffalo.edu
}

Received October 13, 2009; accepted October 14, 2009;

posted November 3, 2009 (Doc. ID 118548); published November 30, 2009

\begin{abstract}
Metamaterials constitute a new area of science that is expanding our fundamental understanding of the behavior of the propagation of electromagnetic waves and their interactions, and providing new solutions for a wide range of applications from optical communications and defense to biological imaging. In this brief review, we focus on recent progress in theoretical, numerical, and experimental studies of linear and nonlinear optical properties of negative index materials and in the emerging field of transformation optics. (C) 2009 Optical Society of America

OCIS codes: $160.3918,250.5403,190.0190$.
\end{abstract}

\section{INTRODUCTION}

Some forty years ago Victor Veselago theoretically investigated a fundamental question of whether the refractive index of a material can take negative values, and predicted a number of unusual phenomena associated with such negative index materials (NIMs) [1]. While NIMs have not been found in nature, in the past decade they were demonstrated first in the microwave and terahertz $[2,3]$ and then in the near-infrared (IR) [4,5] frequency ranges using the so-called metamaterial approach.

Nowadays, the notion of metamaterials embraces a wide range of engineered materials with a unit cell that is much smaller than the wavelength and with predesigned electromagnetic properties, while NIMs are a subclass of metamaterials with the remarkable property of having an antiparallel phase velocity and Poynting vector. To date, a majority of NIMs experimentally demonstrated at visible and near-IR frequencies utilize metallic nanostructures with resonant magnetic response, so that both dielectric permittivity $\epsilon$ and magnetic permeability $\mu$ are negative in the same frequency range [4-10]. While these first experiments proved the possibility of the realization of NIMs, several challenges are still to be solved to make them useful for practical applications. In particular, in the optical frequency range these include (1) further shift of the NIMs operating wavelength to shorter (visible) wavelengths, (2) loss reduction and tunability, and (3) the realization of bulk and broadband NIMs.

Recent progress in fabrication, design, and optimization of metamaterial structures aimed at addressing some of these issues and will be reviewed in Section 2. In Section 3 , some unique nonlinear properties of optical metamaterials are reviewed. Since numerical simulations are an essential component of metamaterial research, in Section 4 we discuss several new developments in numerical modeling of linear and nonlinear, passive and active metamaterials. Finally, in Section 5, we highlight recent advancements in the emerging field of graded-index metamaterials and transformation optics.

\section{OPTICAL NEGATIVE INDEX MATERIALS}

While the first plasmonic NIMs were realized based on a combination of electric and magnetic resonances, it is typically very difficult to obtain a system where both resonances occur at the same frequency, and also, any plasmonic resonance always increases the loss in the system. Moreover, resonant electric response is not really necessary to achieve negative $\epsilon$ since the inherently large negative dielectric permittivity of, for example, noble metals at optical frequencies can be used instead. By combining noble metals with a positive $\epsilon$ dielectric in the proper proportion, any value of $\epsilon$ could be achieved. Therefore, such a nonresonant electric response can be combined with a magnetic resonance to create a negative index of refraction. This general idea was realized in a so-called "fishnet" metamaterial structure that has become a predominant plasmonic NIM geometry (shown in the inset in Fig. 1). Using this structure, significant progress has recently been made towards the realization of negative index of refraction at visible wavelengths. The key milestones in the fabrication of two-dimensional (thin-film) plasmonic metamaterials are summarized in Fig. 1, where $n^{\prime}$ is the real part of the refractive index and $F=\left|n^{\prime}\right| / n^{\prime \prime}$ is a figure of merit, and $n^{\prime \prime}$ is the imaginary part of refractive index [4-10].

Although many unique physical properties can readily be demonstrated in two-dimensional metamaterials, some practical applications require bulk structures. Therefore, considerable efforts have been made in the area of fabrication of three-dimensional metamaterials at optical frequencies [11-13]. Two examples of fabricated structures are shown in Fig. 2.

A major drawback of the above approach, however, is significant losses inevitable in these resonant structures. Indeed, as shown in Fig. 1, the best figure of merit achieved to date is only $\sim 3$. Losses in plasmonic metamaterials have various origins and are still not completely quantified. Some known sources of loss originate from surface roughness, quantum size and chemical interface 


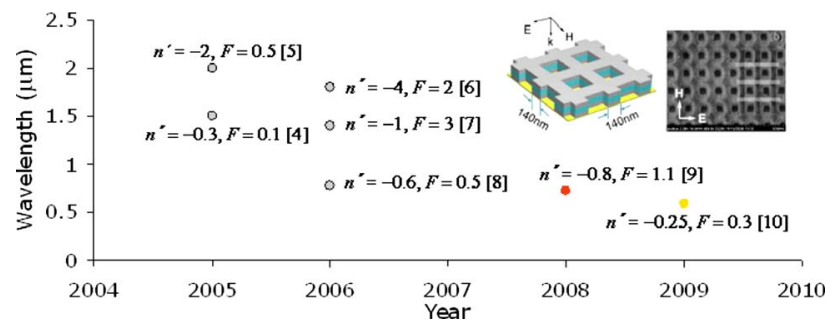

Fig. 1. (Color online) Optical negative-index materials timeline.

effects, the resonant nature of their magnetic response, or the fundamental loss properties of metals. Therefore, several methods have been proposed to reduce or compensate the losses in metamaterials, including incorporation of gain media into the metamaterial design [14-16] and optical-parametric amplification [17-23] discussed in Section 3. Significant, even complete compensation of absorption losses and even lasing was predicted theoretically and demonstrated experimentally in various plasmonic systems [24-38].

Theoretical estimations of the necessary gain levels and initial numerical simulations of realistic losscompensated NIMs have been performed by Klar et al. [15] who proposed immersing the NIM comprising of the double silver strips into the gain medium. It was found that the structure becomes transparent at a gain level of $12 \cdot 10^{3} \mathrm{~cm}^{-1}$. The required amount of gain can be achieved by applying dye molecules such as rhodamine $6 \mathrm{G}$ or semiconductor quantum dots such as CdSe on top of the NIM. Also, recent studies suggested that placing the gain material between the metal strips significantly reduces the required gain because of the enhanced local fields in that area [16].

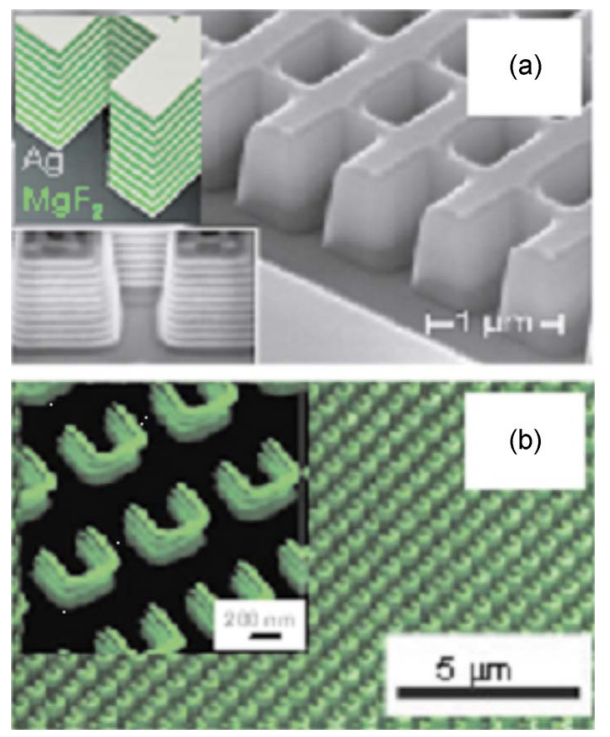

Fig. 2. (Color online) (a) Diagram and SEM image of the 21layer fishnet structure consisting of alternating layers of silver $(\mathrm{Ag})$ and magnesium fluoride $\left(\mathrm{MgF}_{2}\right)$, adapted from [12]. (b) Field-emission scanning electron microscopy images of the fourlayer split-ring resonator structure [13].
An alternative solution to the realization of low-loss NIMs is the development of nonresonant and nonmagnetic approaches to NIMs. One such approach is based on the fact that in a special case of anisotropic materials showing axial symmetry, uniaxially anisotropic materials produce, under certain conditions, antiparallel phase and energy velocity [39-43]. Very recently, the first proof-ofprinciple experimental demonstrations of negative refraction and subdiffraction negative and positive index modes in strongly anisotropic semiconductor waveguides have been reported at mid-IR frequencies [44-46].

The basic idea behind this approach can be understood as follows. Figure 3 shows isofrequency curves and relative directions of the vectors $\mathrm{S}$ and $\mathrm{k}$ for a lossless isotropic medium and for the two cases of uniaxial anisotropy $\left(\epsilon_{x}, \epsilon_{z}>0\right.$ and $\left.\epsilon_{x}<0, \epsilon_{z}>0\right)$. In the anisotropic case the optical axis is pointing along the $x$ axis. Since ordinary waves are not affected by the anisotropy, only extraordinary polarization is considered. In the isotropic case, the wave vector surfaces are circles, and therefore $\mathrm{S}$ $\propto \nabla_{k} \omega(\mathrm{k}) \propto \mathrm{k}$, i.e., vectors $\mathrm{S}$ and $\mathrm{k}$ are collinear [Fig. 3(a)]. For $\epsilon_{x} \neq \epsilon_{z}, \epsilon_{x}, \epsilon_{z}>0$, the wave vector surfaces become ellipsoidal and, as a result, the angle between $\mathrm{S}$ and $\mathrm{k}$ is nonzero [Fig. 3(b)]. Finally, for a material with negative transverse dielectric permittivity $\epsilon_{x}<0$ and positive inplane permittivity $\epsilon_{z}>0$, the dispersion relation becomes hyperbolic. The curvature of the hyperbola is such that the signs of $\mathrm{S}_{z}$ and $\mathrm{k}_{z}$ are opposite [Fig. 3(c)].
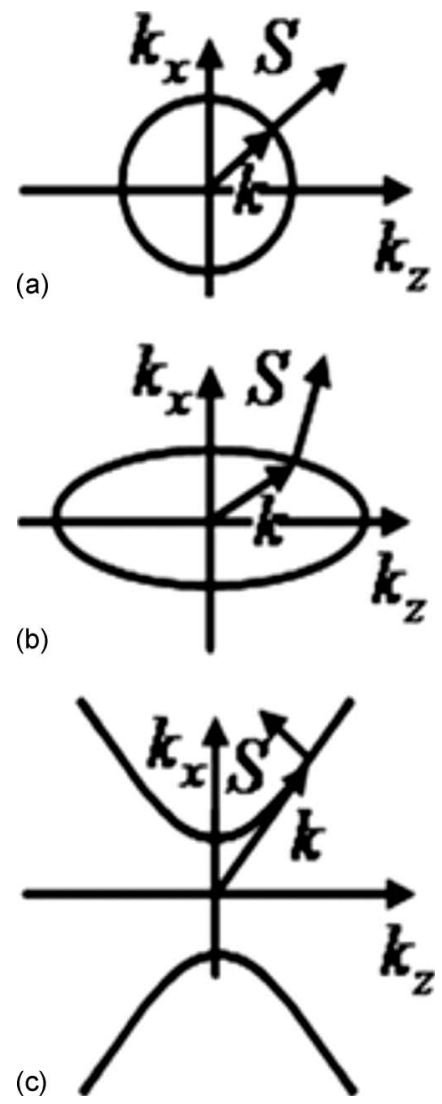

Fig. 3. Isofrequency curves and relative directions of the wave vector $\mathrm{k}$ and the Poynting vector $\mathrm{S}$ for (a) isotropic material, (b) material with $\epsilon_{x}, \epsilon_{z}>0$, (c) material with $\epsilon_{x}<0, \epsilon_{z}>0$ [43]. 
The $z$-component of the Poynting vector for the extraordinary wave can be written as

$$
\mathrm{S}_{z}=\frac{\mathrm{k}_{z}}{2 \omega \epsilon_{x}} H^{2} .
$$

Therefore, if $\epsilon_{x}<0, \mathrm{~S}_{z}$ is also negative, and therefore, $\mathrm{S}_{z}$ is opposite to the direction of the wave vector component $\mathrm{k}_{z}$. This opposite directionality leads to a (effective) negative refractive index of refraction. In particular, an effective negative refractive index has been predicted in a waveguiding system when anisotropic material with $\epsilon_{x}$ $<0$ and $\epsilon_{z}>0$ is used as a core of a planar (in the $y-z$ plane) waveguide with metal walls. In this case negative group velocity modes arise; for these modes the wave vector and the energy flux are antiparallel. In summary, strongly anisotropic waveguides possess the most important characteristic of NIMs-antiparallel directions of wave vector and Poynting vector, and have a potential for the realization of low-loss metamaterials.

Finally, to take full advantage of the refractive index engineering in metamaterials, a method to tune the electromagnetic response of the metamaterial is required. The very first designs of tunable optical metamaterials were based on changing the dielectric function of the nematic liquid crystals using linear effects, either using DC voltage or thermal sources $[47,48]$. Recently, a new approach that utilizes the strong Kerr nonlinearity of the nematic liquid crystals $\left(n_{2} \sim 10^{-2} \mathrm{~cm}^{2} / \mathrm{W}\right)$ to control the refractive index in optical metamaterials was investigated [49]. Numerical simulations predicted transmission minimum shifts comparable to the relative changes induced previously in metamaterials in the microwave range. Moreover, since the new approach employs the significant near-field enhancement of the electric field, and thus, the localized nonlinear response, the resulting optically tunable shift is larger than the shift induced with a uniform linear bias-field of a similar magnitude. One of the limitations of this approach is the relatively slow nonlinear response of the liquid crystals. In order to overcome this limitation, semiconductor nonlinearities with picosecond response time could potentially be explored.

\section{NONLINEAR OPTICS IN METAMATERIALS}

Metamaterials necessitate the reconsideration of many well-known nonlinear optical phenomena, including harmonic generation, parametric processes, optical bistability, modulational instability, and soliton propagation [17-23,50-62]. One of the most remarkable properties enabled by antiparallel wave and Poynting vectors in NIMs is a novel, backward phase-matching mechanism. Backward phase-matching in combination with the strong frequency dependence of the metamaterial parameters was shown to facilitate new regimes of second-harmonic generation (SHG) [17,53] and optical parametric amplification (OPA) [17-23].

The basic idea of the backward phase-matching mechanism can be described as follows. For example, let's assume that the metamaterial is a NIM at the fundamental frequency $\omega_{1}$ and it is a positive-index material (PIM) at the second-harmonic frequency $\omega_{2}$. If the energy flow of the fundamental frequency travels from left to right, the phase of the wave at the same frequency should move in the opposite direction, that is, from right to left. The phase-matching requirement $\mathrm{k}_{2}=2 \mathrm{k}_{1}$ can be satisfied if the phase of the second harmonic also travels from right to left. Since the second harmonic propagates in the PIM, its energy flow is co-directed with the phase velocity and, therefore, the energy propagates from right to left as well. On the contrary, in conventional PIM materials, the wave and Poynting vectors propagate in the same direction at both the fundamental and second-harmonic frequency and backward phase-matching generally does not occur.

One of the important differences between the SHG in the NIM and PIM cases is reflected in the Manley-Rowe relations given by $\left|A_{1}\right|^{2}-\left|A_{2}\right|^{2}=C$, where $A_{1}$ and $A_{2}$ are slowly varying amplitudes of the fundamental and second-harmonic waves, respectively. In the conventional PIM case, the Manley-Rowe relations require that the sum of the squared amplitudes is constant; however, in the NIM case their difference is constant. It is noteworthy that similar relations are typical to distributed feedback structures; however, in the case of metamaterials the effective feedback is facilitated by the NIM-PIM structure itself. In the limit of a semi-infinite NIM, both the fundamental and second-harmonic waves disappear at infinity. Therefore, a $100 \%$ conversion efficiency of the incoming wave at the fundamental frequency to the secondharmonic frequency propagating in the opposite direction is expected. As a result, the NIM slab acts as a nonlinear mirror.

An important potential application of backward phasematching realized in NIMs is the compensation of losses. Recently, a new approach to overcome losses in metamaterials over the entire negative-index frequency range was proposed [20]. The proposed technique is based on a well-known three-wave mixing process that takes place in nonlinear media exhibiting second-order susceptibility (in $\chi^{(2)}$ materials), and induces optical parametric amplification. In optical parametric amplification, the photon flux in the signal wave grows through coherent energy transfer from a higher-frequency strong pump wave. A photon from an incident pump laser is divided into two photons, one of which is a photon at the signal frequency. The strong pump field with angular frequency and wavenumber $\left(\omega_{3}, \mathrm{k}_{3}\right)$ and a weak signal with $\left(\omega_{1}, \mathrm{k}_{1}\right)$ generate a difference frequency idler with $\left(\omega_{2}, \mathrm{k}_{2}\right)$. The OPA process requires both momentum and energy conservation. Momentum is conserved when the phase-matching condition for the wavevectors, $\mathrm{k}_{3}=\mathrm{k}_{1}+\mathrm{k}_{2}$ is satisfied, whereas energy conservation requires that $\omega_{3}=\omega_{1}+\omega_{2}$.

Metamaterials represent a unique environment for the realization of a backward OPA. Indeed, an OPA with counter-directed energy flows can be realized with all three waves having co-directed wave vectors, as shown in Fig. 4(a). Indeed, if the pump and idler wave propagate in the positive-index regime, and the signal wave frequency belongs to the negative-index regime, the energy flow of the signal wave will be antiparallel to that of the pump and the idler. This will create an effective feedback mechanism. Such a NIM-based OPA was shown theoretically to exhibit peculiar oscillatory distributions of the 

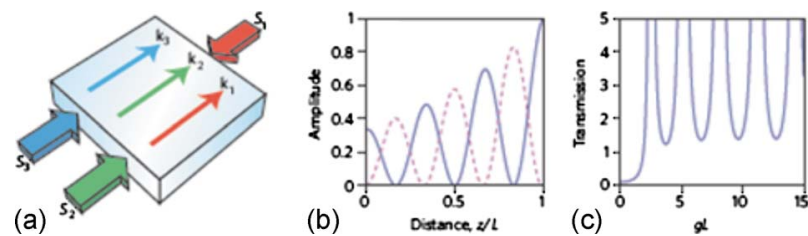

Fig. 4. (Color online) Optical parametric amplification in NIMs. (a) Schematic representation of backward OPA in a NIM slab. $S_{1}$, $S_{2}$ and $S_{3}$ are the magnitudes of the Poynting vectors of the signal, idler and pump beam, respectively, and $\mathrm{k}_{1}, \mathrm{k}_{2}$ and $\mathrm{k}_{3}$ are their wavevectors. (b) Signal (solid curve) and idler (dashed curve) field distributions inside the NIM. All waves propagate in the $z$-direction and the signal enters NIM at $z=L$, where $L$ is the slab thickness. (c) Signal amplification in the NIM slab. $g$ is a nonlinear coupling coefficient [21].

fields shown in Fig. 4(b). As a result, the scheme supports oscillations without a cavity as each spatial point serves as a source for the generated wave in the backward direction.

Another remarkable property of such a NIM-based OPA, illustrated in Fig. 4(c), is that the originally lossy NIM becomes transparent (or even amplifying) for a broad range of pump field intensities and slab thicknesses, provided that the absorption (loss) coefficient at the idler frequency is equal to or larger than that at the signal frequency. A significant advantage of the OPA approach as compared to other loss-compensating techniques is that it is not limited to the narrow wavelength range defined by the laser transitions of the laser gain media such as rhodamine $6 \mathrm{G}$ or quantum dots and, therefore, can be made tunable in a wide frequency range.

\section{NUMERICAL MODELING AND OPTIMIZATION METHODS}

Due to the complexity of metamaterial structures, numerical simulations are of paramount importance in metamaterial research. Conventional modeling tools have to be tailored, and new tools need to be developed to account for the subwavelength and dispersive nature of metamaterial structures, singularities, and instabilities caused by high-index contrast, the presence of metals, and interfaces between positive- and negative-refractive index materials, and an extremely large number of unit cells. Finally, software developed for modeling conventional optical materials and devices usually does not take into account any magnetic properties, as it is commonly assumed that $\mu \approx 1$ at optical frequencies.

Although the field of optical metamaterials is relatively new, there are a number of numerical approaches and tools developed for design, optimization, characterization, and modeling of linear and nonlinear wave propagation in metamaterials. In this section, we review some of these newly developed methods.

The typical design of metamaterials starts with the unit cell, such as a pair of nanorods, coupled strips, or a fishnet structure. The dimensions of these unit cells and the material properties form a hyperspace for the global optimization problem [63]. Several optimization methods have been developed and applied to designing of metamaterials, including: (1) simulated annealing [63,64], (2) genetic algorithms [65], and (3) particle swarm optimization
[66]. The efficient design procedures incorporate realistic fabrication constraints, constraints on material properties that can be employed, and an understanding of the fabrication process itself.

An important aspect of realistic metamaterial design is related to the fact that plasmonic structures are typically very lossy. Therefore, the realization of the exceptional electromagnetic properties and novel functionalities associated with metamaterials requires a significant reduction of their losses. Recently, a new self-consistent, frequency-domain model of a gain system for the study of loss compensation in negative-index materials (NIMs) in a pump-probe configuration was proposed [16]. While previously developed approaches assumed a certain pumpfield distribution rather than solving the actual nonlinear problem, generally, gain systems are described by a saturable absorptive response, and therefore, they are inherently nonlinear. As a result, the gain induced by the pump light may not be uniform in space. The pump-probe configuration requires a two-step solution: The first step involves the solution of the nonlinear equations for the pump; the second stage of the solution process involves solving the equations governing the probe light distribution. Using this approach, it was shown that complete compensation can be obtained for emitters with realistic gain coefficients if they are incorporated into the spacer layer where the field enhancement is maximal [16].

In the past few years, a number of unusual nonlinear wave interactions were predicted to occur in metamaterials. In a majority of theoretical studies to date, nonlinear metamaterials were considered as uniform media with prescribed dielectric permittivity and magnetic permeability, and the nanostructured nature of these artificial materials was not taken into account. Local field enhancement and other effects are likely to alter nonlinear interactions of electromagnetic waves with metamaterials. Therefore, availability of efficient and reliable numerical modeling tools that take into account actual nanostructure and near-field effects is essential for developing future nonlinear applications of metamaterials.

Recently, an efficient method for introducing thirdorder nonlinearities in optical nanostructured materials, including photonic metamaterials, was developed $[67,68]$. While a standard TM representation using cubic nonlinear susceptibility is problematic due to an intractable implicit equation, the new method uses scalar H-field frequency domain formulation. It was shown to produce fast and accurate results without superfluous vector E-field formalism. The 2D H-field formulation was found to exhibit a substantially faster performance and converged over a broader range of nonlinearities compared with the 3D formulation.

\section{GRADIENT-INDEX AND TRANSFORMATION OPTICS}

Metamaterial technology opens nearly unlimited opportunities in refractive index engineering, offering exceptional flexibility with respect to the profile, depth, and absolute value of the refractive index change, and precise control over its distribution, enabling unprecedented opportunities for light manipulation. 
It is worth noting that the most unusual properties of NIMs are revealed at the interface of positive- and negative-index materials. Particularly, the right-handed triplet formed from the electric and magnetic fields and the wave vector in the PIM undergoes an abrupt change to form a left-handed triplet in the NIM. A topologically critical phenomenon such as this leads to antiparallel directions of the wave and Poynting vectors in the NIM.

Until recently, most of the studies have been focused on abrupt transitions from PIMs to NIMs in homogeneous structures. However, recently it was realized that a number of remarkable phenomena occurs in metamaterials with material properties gradually changing from positive to negative values, or so-called as transition metamaterials [69-71]. In particular, it was shown that in the case of a smooth (gradual) refractive index change from positive to negative values, as illustrated in Fig. 5 upper plot), one can observe the resonant field enhancement of the $H_{x}$ component near the zero-refractive-index point under oblique incidence of the TE wave in such a gradientindex metamaterial layer. In a simplified way, the origin of the anomalous field enhancement shown in Fig. 5 lower plot) can be understood as a spatial analog of the wellknown resonance occurring in the spectral domain when, for example, light interacts with a harmonic oscillator. It is noteworthy that, because the wavelength of light becomes very large in the vicinity of the zero-index point, the system can effectively be considered as static-like. In the TE wave case, the magnetic field has a nonzero component in the direction of propagation, and the magnetic field energy accumulates in the vicinity of the zero-index point in space. Such a thin layer near the zero-index point can be considered to be a short solenoid that stores the magnetic field energy. In this case, $H$ anomalously in-
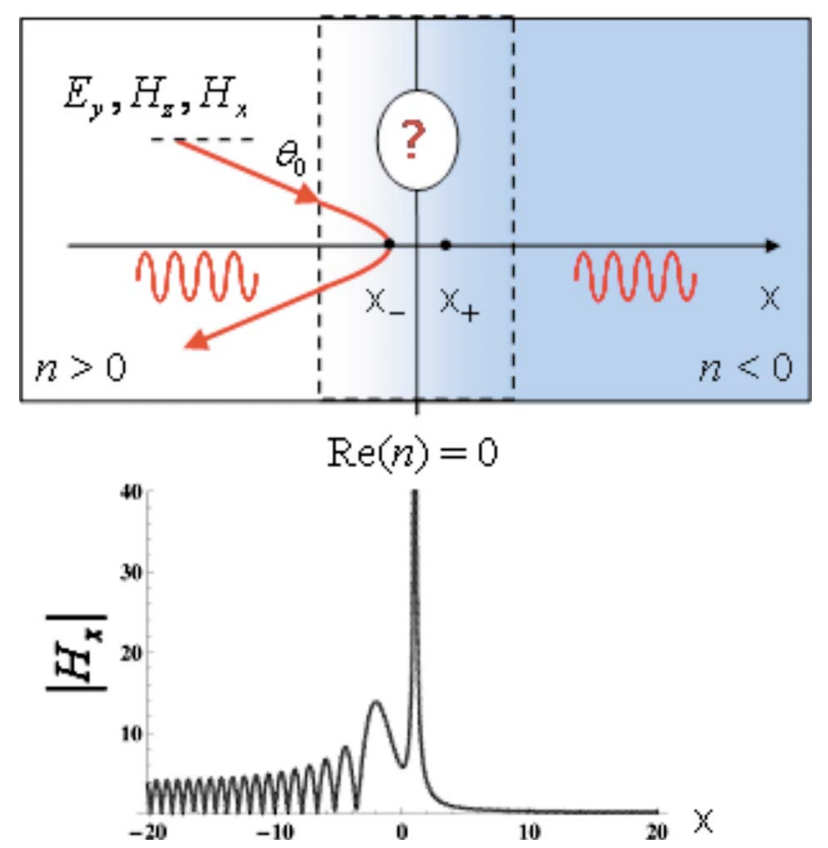

Fig. 5. (Color online) The real parts of the dielectric permittivity and magnetic susceptibility as functions of a longitudinal coordinate (upper plot), and the absolute value of the normalized magnetic field component as a function of normalized longitudinal (lower plot). creases as the refractive index tends to zero. Finally, owing to the singularities of the magnetic field components, the $x$ and $z$ components of the Poynting vector are also singular at the zero-index point. These findings may enable a variety of applications in microwave, terahertz, and optical metamaterials, including subwavelength transmission, wave concentrators, and low-intensity nonlinear optical devices.

The enormous potential of refractive-index engineering in metamaterial structures can be further exemplified by recent progress in the field of transformation optics that enabled novel opportunities in the design of graded-index structures [72-84]. First applied to the development of the cloak, transformation method is now considered a very general and powerful design tool that offers unparalleled opportunities for controlling light propagation through careful refractive-index engineering.

The basic idea of transformation method [72,85,86] is that, in order to guide waves along a certain trajectory, either the space should be deformed, assuming that material properties remain the same, or the material properties should be properly modified. The latter approach is usually employed. More specifically, under a coordinate transformation, the form of Maxwell's equations should remain invariant while new $\epsilon$ and $\mu$ would contain the information regarding the coordinate transformation and the original material parameters. Therefore, in order to design a particular property/light trajectory, it is possible to design an anisotropic material with prescribed components of permittivity and permeability tensors calculated through a particular coordinate transformation.

The general design approach using the transformation method includes two main steps. In the first step, a coordinate transformation of the space with the desired property is built. In the next step, a set of material properties is calculated that would realize this property of the transformed space in the original space using the following equations:

$$
\begin{aligned}
& \epsilon^{i^{\prime} j^{\prime}}=\left|\operatorname{det}\left(\Lambda_{i}^{i^{\prime}}\right)\right|^{-1} \Lambda_{i}^{i^{\prime}} \Lambda_{j}^{j^{\prime}} \epsilon, \\
& \mu^{i^{\prime} j^{\prime}}=\left|\operatorname{det}\left(\Lambda_{i}^{i^{\prime}}\right)\right|^{-1} \Lambda_{i}^{i^{\prime}} \Lambda_{j}^{j^{\prime}} \mu,
\end{aligned}
$$

where it was assumed that the original space is isotopic, transformations are time invariant, and $\Lambda_{\alpha}^{\alpha^{\prime}}=\partial x^{\alpha^{\prime}} / \partial x^{\alpha}$ are the elements of the Jacobian transformation matrix.

Recently, various functionalities and novel device applications enabled by this approach have been proposed, including light concentrators [78], lenses [79], gradientindex waveguides and bends [80,81], black holes [82], and even illusion devices [83]. Some of these devices are illustrated in Fig. 6. Unfortunately, many of these devices, including the first cloaking devices, were inherently narrowband. Indeed, since the refractive index of the cloaking shell was designed to vary from zero to one, the phase velocity of light inside the shell is greater than the velocity of light in a vacuum. While this condition itself does not contradict any law of physics, it implies that the material parameters must be dispersive. Therefore, one of the remaining challenges is the realization of broadband transformation-optics-based applications.

To address this challenge, the transformation method was applied to design a broadband cloaking device that 


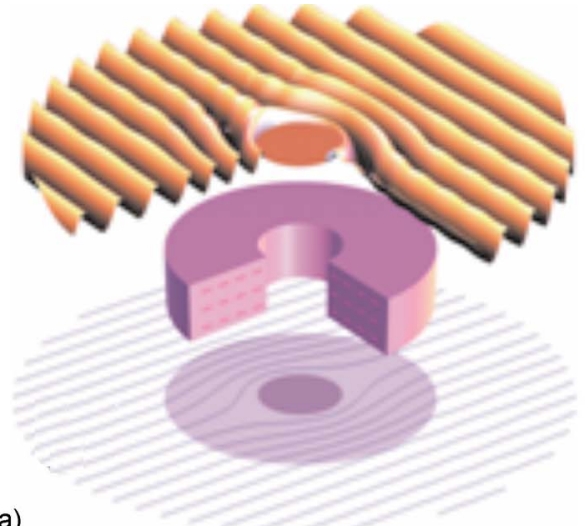

(a)

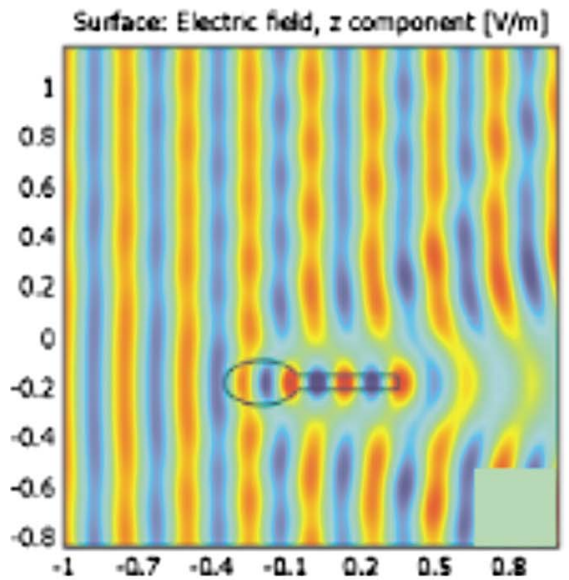

(c)

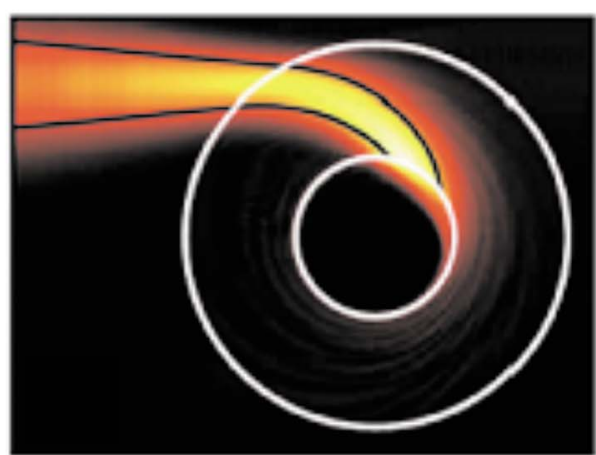

(b)

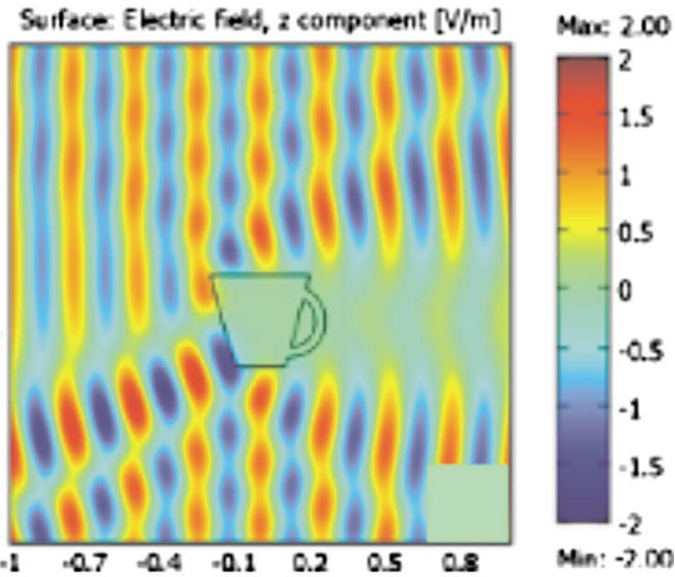

(d)

Fig. 6. (Color online) Examples of gradient-index structures designed using the transformation optics approach.

functions in a wavelength multiplexing manner shown in Fig. 7 [84]. The basic idea can be understood as follows: Since the anisotropic constituent materials of a cloak for one wavelength cannot be transparent at other frequencies, cloaks for all the wavelengths being considered have to share the same outer boundary-the physical boundary of the device. The inner boundary and the transformation for each operating wavelength, however, are unique, as shown in Figs. 7(a)-7(c).

Recently, two other, very different designs of optical cloaks that address the narrowband issue were proposed and experimentally demonstrated. One of them is a socalled carpet cloak that is made of a dielectric and operates in a broad range of wavelengths [87-90]. The basic idea behind this approach is illustrated in Fig. 8(a). The carpet cloak compresses an object in only one direction into a conducting sheet. When the object is placed under a curved reflecting surface with the carpet cloak on top of it, the object appears as if it was the original flat reflecting surface, so it is hidden under a "carpet." This approach avoids both material and geometry singularities. The
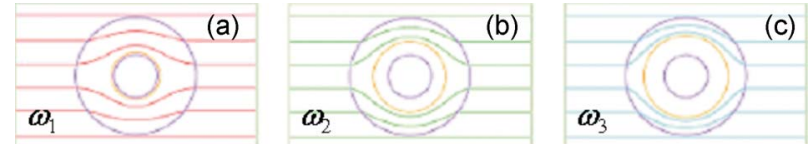

Fig. 7. (Color online) Simulated schematic of a cloaking system for multiple wavelengths or a finite bandwidth, with $\omega_{1}>\omega_{2}$ $>\omega_{3}$, shown in (a), (b), and (c), respectively [84].
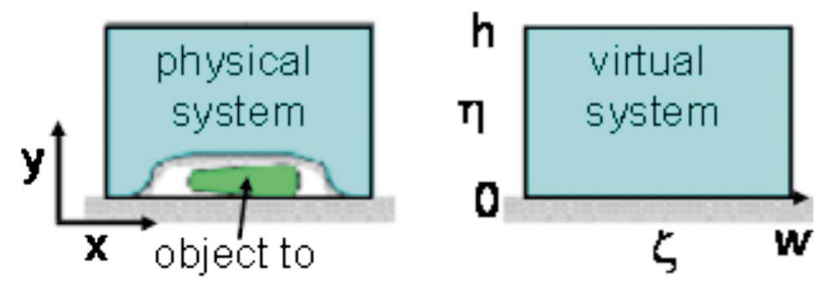

(a)

conceal
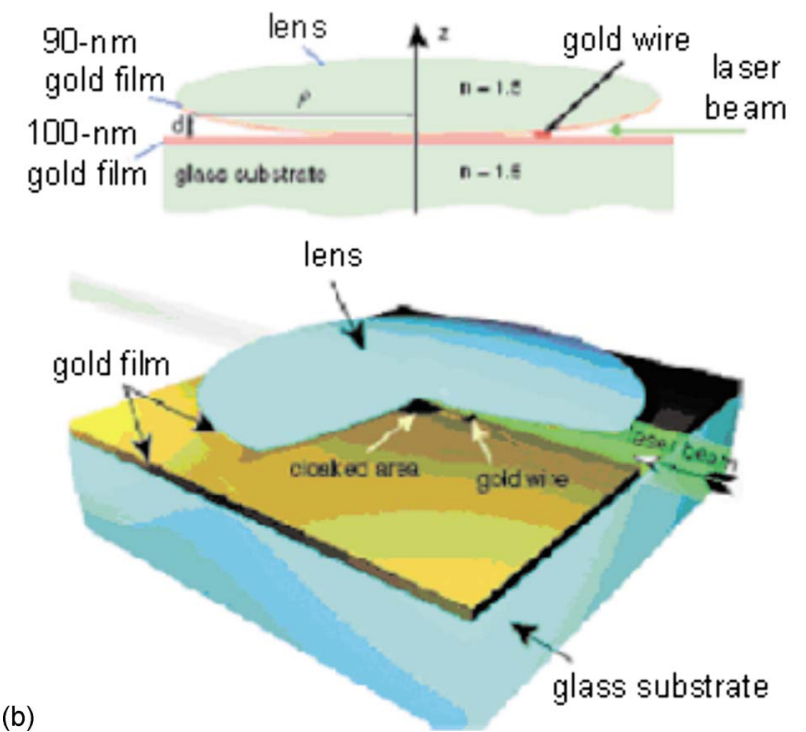

Fig. 8. (Color online) (a) Optical carpet cloaking [87]. (b) Tapered waveguide acting as an optical cloak [91]. 
cloak region is obtained by varying the effective refractive index in a $2 \mathrm{D}$ space. This index profile is designed using quasi-conformal mapping. The carpet-cloaking approach solves several problems associated with other approaches. The approach can be implemented using nonresonant elements (e.g., conventional dielectric materials) and, therefore allows for achieving low-loss and broadband cloaking at optical wavelengths. Indeed, this approach was already demonstrated in a broad range of optical wavelengths extending from 14 to $1800 \mathrm{~nm}$.

Finally, very recently another extremely simple approach to cloaking was proposed and experimentally demonstrated. In this approach, the metamaterial requiring anisotropic dielectric permittivity and magnetic permeability is emulated by a specially designed, tapered waveguide [91]. It was shown that the transformation optics approach allows us to map a planar region of space filled with an inhomogeneous, anisotropic metamaterial into an equivalent region of empty space with curvilinear boundaries (a tapered waveguide).

This approach leads to low-loss, broadband performance in the visible wavelength range. A schematic of the cloak is shown in Fig. 8(b). A double convex glass lens coated on one side with a gold film is placed with the goldcoated side down on top of a flat gold-coated glass slide. The air gap between these surfaces can be used as an adiabatically changing waveguide. In fact, this geometry is identical to the classic geometry of the Newton rings observation.

It is well known that the modes of the waveguide have cutoff wavelengths. It turns out that, for a particular mode in the waveguide of Fig. 8(b), the cutoff radius is given by the same expression as that of the radius of the corresponding Newton ring; thus, no photon launched into the waveguide can reach an area within the radius from the point of contact between the two gold-coated surfaces. This approach leads to low-loss, broadband cloaking performance. Importantly, the cloak that has already been realized with this approach was one-hundred times larger than the wavelength of light, a property that was not demonstrated with any other cloaking techniques to date.

\section{SUMMARY}

In summary, over the past ten years, enormous progress in the field of metamaterials has been made. As a result, fascinating theoretical predictions were transformed into an even more amazing reality of materials with properties far exceeding those available in nature. These new material properties and device functionalities enabled by metamaterials technology have a strong potential for contributing to nearly all areas of fundamental and applied science and technology, including imaging, defense, telecommunications, optical computing, and sensing.

\section{ACKNOWLEDGMENTS}

The authors acknowledge the support of the U.S. Army Research Office (USARO) through grants W911NF-09-10075, W911NF-09-1-0231, and 50342-PH-MUR.

\section{REFERENCES}

1. V. G. Veselago, "The electrodynamics of substances with simultaneously negative values of $\epsilon$ and $\mu$," Sov. Phys. Usp. 10, 509-514 (1968).

2. D. R. Smith, W. J. Padilla, D. C. Vier, S. C. Nemat-Nasser, and S. Schultz, "Composite medium with simultaneously negative permeability and permittivity," Phys. Rev. Lett. 84, 4184-4187 (2000)

3. S. Linden, C. Enkrich, M. Wegener, J. Zhou, T. Koschny, and C. M. Soukoulis, "Magnetic response of metamaterials at 100 terahertz," Science 306, 1351-1353 (2004).

4. V. M. Shalaev, W. Cai, U. K. Chettiar, H. Yuan, A. K. Sarychev, V. P. Drachev, and A. V. Kildishev, "Negative index of refraction in optical metamaterials," Opt. Lett. 30, 3356-3358 (2005).

5. S. Zhang, W. Fan, N. C. Panoiu, K. J. Malloy, R. M. Osgood, and S. R. J. Brueck, "Experimental demonstration of nearinfrared negative-index metamaterials," Phys. Rev. Lett. 95, 137404 (2005).

6. S. Zhang, W. Fan, K. J. Malloy, S. R. J. Brueck, N. C. Panoiu, and R. M. Osgood, "Demonstration of metaldielectric negative-index metamaterials with improved performance at optical frequencies," J. Opt. Soc. Am. B 23, 434-438 (2006).

7. G. Dolling, C. Enkrich, M. Wegener, C. M. Soukoulis, and S. Linden, "Low-loss negative-index metamaterial at telecommunication wavelengths," Opt. Lett. 31, 1800-1802 (2006).

8. G. Dolling, M. Wegener, C. M. Soukoulis, and S. Linden, "Negative-index metamaterial at $780 \mathrm{~nm}$ wavelength," Opt. Lett. 32, 53-55 (2007).

9. U. K. Chettiar, S. Xiao, A. V. Kildishev, W. Cai, H.-K. Yuan, V. P. Drachev, and V. M. Shalaev, "Optical metamagnetism and negative-index metamaterials," MRS Bull. 33, 921-926 (2008).

10. S. Xiao, U. K. Chettiar, A. V. Kildishev, V. P. Drachev, and V. M. Shalaev, "Yellow-light negative-index metamaterials," Opt. Lett. 34, 3478-3480 (2009).

11. G. Dolling, M. Wegener, and S. Linden, "Realization of a three-functional-layer negative-index photonic metamaterial," Opt. Lett. 32, 551-553 (2007).

12. J. Valentine, S. Zhang, T. Zentgraf, E. Ulin-Avila, D. A. Genov, G. Bartal, and X. Zhang, "Three-dimensional optical metamaterial with a negative refractive index," Nature 455, 376-379 (2008).

13. N. Liu, H. Guo, L. Fu, S. Kaiser, H. Schweizer, and H. Giessen, "Three-dimensional photonic metamaterials at optical frequencies," Nature Mater. 7, 31-37 (2008).

14. S. A. Ramakrishna and J. B. Pendry, "Removal of absorption and increase in resolution in a near-field lens via optical gain," Phys. Rev. B 67, 201101 (2003).

15. T. A. Klar, A. V. Kildishev, V. P. Drachev, and V. M. Shalaev, "Negative-index materials: going optical," IEEE J. Sel. Top. Quantum Electron. 12, 1106-1115 (2006).

16. Y. Sivan, S. Xiao, U. K. Chettiar, A. V. Kildishev, and V. M. Shalaev, "Frequency-domain simulations of a negativeindex material with embedded gain," Opt. Express 17, in press (2009).

17. A. K. Popov and V. M. Shalaev, "Negative-index metamaterials: second-harmonic generation, Manley-Rowe relations and parametric amplifications," Appl. Phys. B: Photophys. Laser Chem. 84, 131-137 (2006).

18. A. K. Popov and V. M. Shalaev, "Compensating losses in negative-index metamaterials by optical parametric amplification,” Opt. Lett. 31, 2169-2171 (2006).

19. A. K. Popov, S. A. Myslivets, T. F. George, and V. M. Shalaev, "Four-wave mixing, quantum control and compensating losses in doped negative-index photonic metamaterials," Opt. Lett. 32, 3044-3046 (2007).

20. A. K. Popov and S. A. Myslivets, "Transformable broadband transparency and amplification in negative-index films," Appl. Phys. Lett. 93, 191117 (2008).

21. N. M. Litchinitser and V. M. Shalaev, "Loss as a route to transparency," Nature Photon. 3, 75-76 (2009).

22. A. K. Popov, S. A. Myslivets, and V. M. Shalaev, 
"Microscopic mirrorless negative-index optical parametric oscillator," Opt. Lett. 34, 1165-1167 (2009).

23. A. K. Popov, S. A. Myslivets, and V. M. Shalaev, "Resonant nonlinear optics of backward waves in negative-index metamaterials," Appl. Phys. B: Photophys. Laser Chem. 96, 315-323 (2009).

24. N. Sudarkin and P. A. Demkovich, "Excitation of surface electromagnetic waves on the boundary of a metal with an amplifying medium," Sov. Phys. Tech. Phys. 34, 764-766 (1989).

25. M. P. Nezhad, K. Tetz, and Y. Fainman, "Gain assisted propagation of surface plasmon polaritons on planar metallic surfaces," Opt. Express 12, 4072-4079 (2004).

26. S. A. Maier, "Gain-assisted propagation of electromagnetic energy in subwavelength surface plasmon polariton gap waveguides," Opt. Commun. 258, 295-299 (2006).

27. J. Seidel, S. Grafström, and L. Eng, "Stimulated emission of surface plasmons at the interface between a silver film and an optically pumped dye solution," Phys. Rev. Lett. 94, 177401 (2005).

28. N. M. Lawandy, "Localized surface plasmon singularities in amplifying media," Appl. Phys. Lett. 85, 5040-5042 (2004).

29. I. Avrutzki, "Surface plasmons at nanoscale relief gratings between a metal and a dielectric medium with optical gain," Phys. Rev. B 70, 155416 (2004).

30. M. A. Noginov, G. Zhu, M. Bahoura, J. Adegoke, C. E. Small, B. A. Ritzo, V. P. Drachev, and V. M. Shalaev, "Enhancement of surface plasmon resonance in an $\mathrm{Ag}$ aggregate by optical gain in a dielectric medium," Opt. Lett. 31, 3022-3024 (2006).

31. A. K. Sarychev and G. Tartakovsky, "Magnetic plasmonic metamaterials in actively pumped host medium and plasmonic nanolaser," Phys. Rev. B 75, 085436 (2007).

32. J. A. Gordon and R. Ziolkowsky, "The design and simulated performance of a coated nano-particle laser," Opt. Express 15, 2622-2653 (2007).

33. M. A. Noginov, G. Zhu, M. Mayy, B. A. Ritzo, and V. A. Podolskiy, "Stimulated emission of surface plasmon polaritons," Phys. Rev. Lett. 101, 226806 (2008).

34. N. I. Zheludev, S. L. Prosvirnin, N. Papasimakis, and V. A. Fedotov, "Lasing-spaser," Nature Photon. 2, 351-354 (2008).

35. I. De Leon and P. Berini, "Theory of surface plasmonpolariton amplification in planar structures incorporating dipolar gain media," Phys. Rev. B 78, 161401 (2008).

36. M. Ambati, S. H. Nam, E. Ullin-Avilla, D. A. Genov, G. Bartal, and X. Zhang, "Observation of stimulated emission of surface plasmon polaritons," Nano Lett. 8, 3998-4001 (2008)

37. A. Fang, T. Koschny, M. Wegener, and C. M. Soukoulis, "Self-consistent calculation of metamaterials with gain," Phys. Rev. B 79, 241104 (2009).

38. M. A. Noginov, G. Zhu, A. M. Belgrave, R. Bakker, V. M. Shalaev, E. E. Narimanov, S. Stout, E. Herz, T. Suteewong, and U. Wiesner, "Demonstration of a SPASER-based nanolaser," Nature 460, 1110-1112 (2009).

39. P. A. Belov, "Backward waves and negative refraction in uniaxial dielectrics with negative dielectric permittivity along the anisotropy axis," Microwave Opt. Technol. Lett. 37, 259-263 (2003).

40. V. A. Podolskiy, and E. E. Narimanov, "Strongly anisotropic waveguide as a nonmagnetic left-handed system," Phys. Rev. B 71, 201101 (2005).

41. V. A. Podolskiy, L. Alekseyev, and E. E. Narimanov, "Strongly anisotropic media: the $\mathrm{THz}$ perspectives of lefthanded materials," J. Mod. Opt. 52, 2343-2349 (2005).

42. R. Wangberg, J. Elser, E. E. Narimanov, and V. A. Podolskiy, "Nonmagnetic nanocomposites for optical and infrared negative-refraction-index media," J. Opt. Soc. Am. B 23, 498-505 (2006).

43. L. V. Alekseyev and E. E. Narimanov, "Slow light and 3D imaging with non-magnetic negative index systems," Opt. Express 14, 11184-11193 (2006).

44. A. Hoffman, L. Alekseyev, S. Howard, K. Franz, D. Wisserman, V. Podolskiy, E. Narimanov, D. Sivco, and C. Gmachl, "Negative refraction in semiconductor metama- terials, Nature Mater. 6, 946-950 (2007).

45. A. J. Hoffman, V. A. Podolskiy, D. L. Sivco, and C. Gmachl, "Sub-diffraction negative and positive index modes in midinfrared waveguides," Opt. Express 16, 16404-16409 (2008).

46. A. J. Hoffman, A. Sridhar, P. X. Braun, L. Alekseyev, S. S. Howard, K. J. Franz, L. Cheng, F.-S. Choa, D. L. Sivco, V. A. Podolskiy, E. E. Narimanov, and C. Gmachl, "Midinfrared semiconductor optical metamaterials," J. Appl. Phys. 105, 122411 (2009).

47. X. Wang, D.-H. Kwon, D. H. Werner, I.-C. Khoo, A. V. Kildishev, and V. M. Shalaev, "Tunable optical negativeindex metamaterials employing anisotropic liquid crystals," Appl. Phys. Lett. 91, 143122 (2007).

48. S. Xiao, U. K. Chettiar, A. V. Kildishev, V. Drachev, I. C. Khoo, and V. M. Shalaev, "Tunable magnetic response of metamaterials," Appl. Phys. Lett. 95, 033115 (2009).

49. Y. Sivan, A. V. Kildishev, N. M. Litchinitser, I. C. Khoo, and V. M. Shalaev, "Nonlinear tuning in optical metamaterials," presented at SPIE Optics \& Photonics Conference, Liquid Crystals XIII, San Diego, California, August 2-6, 2009

50. M. W. Feise, I. V. Shadrivov, and Y. S. Kivshar, "Tunable transmission and bistability in left-handed band-gap structures," Appl. Phys. Lett. 85, 1451-1453 (2004).

51. A. D. Boardman, P. Egan, L. Velasco, and N. King, "Control of planar nonlinear guided waves and spatial solitons with a left-handed medium,” J. Opt. A, Pure Appl. Opt. 7, S57-S67 (2005).

52. I. R. Gabitov, R. Indik, N. M. Litchinitser, A. I. Maimistov, V. M. Shalaev, and J. E. Soneson, "Double resonant optical materials with embedded metallic nanostructures," J. Opt. Soc. Am. B 23, 535-542 (2006).

53. A. K. Popov, V. V. Slabko, and V. M. Shalaev, "Second harmonic generation in left-handed metamaterials," Laser Phys. Lett. 3, 293-297 (2006).

54. M. W. Klein, C. Enkrich, M. Wegener, and S. Linden, "Second-harmonic generation from magnetic metamaterials," Science 313, 502-504 (2006).

55. M. Scalora, G. D’Aguanno, M. J. Bloemer, M. Centini, D. de Ceglia, N. Mattiucci, and Y. S. Kivshar, "Dynamics of short pulses and phase matched second harmonic generation in negative index materials," Opt. Express 14, 4746-4756 (2006).

56. D. de Ceglia, A. D’Orazio, M. de Sario, V. Petruzzelli, F. Prudenzano, M. Centini, M. G. Cappeddu, M. J. Bloemer, and M. Scalora, "Enhancement and inhibition of secondharmonic generation and absorption in a negative index cavity," Opt. Lett. 32, 265-267 (2007).

57. N. M. Litchinitser, I. R. Gabitov, and A. I. Maimistov, "Optical bistability in a nonlinear optical coupler with a negative index channel," Phys. Rev. Lett. 99, 113902(4) (2007).

58. N. M. Litchinitser, I. R. Gabitov, A. I. Maimistov, and V. M. Shalaev, "Effect of an optical negative refractive index thin film on optical bistability," Opt. Lett. 32, 151-153 (2007).

59. M. W. Klein, M. Wegener, N. Feth, and S. Linden, "Experiments on second- and third-harmonic generation from magnetic metamaterials," Opt. Express 15, 5238-5247 (2007).

60. M. W. Klein, M. Wegener, N. Feth, and S. Linden, "Experiments on second- and third-harmonic generation from magnetic metamaterials," erratum, Opt. Express 16, 8055 (2008).

61. E. Kim, F. Wang, W. Wu, Z. Yu, and Y. R. Shen, "Nonlinear optical spectroscopy of photonic metamaterials," Phys. Rev. B 78, 113102(4) (2008).

62. A. I. Maimistov, I. R. Gabitov, and N. M. Litchinitser, "Solitary waves in a nonlinear oppositely directed coupler," Opt. Spectrosc. 104, 253-257 (2008).

63. A. V. Kildishev, U. K. Chettiar, Z. Liu, V. M. Shalaev, D.-H. Kwon, Z. Bayraktar, and D. H. Werner, "Stochastic optimization of low-loss optical negative-index metamaterial," J. Opt. Soc. Am. B 24, A34-A39 (2007).

64. D. T. Pham and D. Karaboga, Intelligent Optimization Techniques (Springer-Verlag, 2000). 
65. R. L. Haupt and S. E. Haupt, Practical Genetic Algorithms (Wiley-Interscience, 2004).

66. J. Kennedy and R. C. Eberhart, Swarm Intelligence (Academic, 2001).

67. A. V. Kildishev, Y. Sivan, N. M. Litchinitser, and V. M. Shalaev, "Frequency-domain modeling of scalar TM wave propagation in optical nanostructures with a third-order nonlinear response," Opt. Lett. 34, 3364-3366 (2009).

68. A. V. Kildishev and N. M. Litchinitser, "Efficient simulation of non-linear effects in 2D optical nanostructures to TM waves," Opt. Commun. Doi:10.1016/j.optcom.2009.09.039.

69. N. M. Litchinitser, A. I. Maimistov, I. R. Gabitov, R. Z Sagdeev, and V. M. Shalaev, "Metamaterials: electromagnetic enhancement at zero-index transition," Opt. Lett. 33, 2350-2352 (2008).

70. M. Dalarsson and P. Tassin, "Analytical solution for wave propagation through a graded index interface between a right-handed and a left-handed material," Opt. Express 17, 6747-6752 (2009)

71. K. Kim, D.-H. Lee, and H. Lim, "Resonant absorption and mode conversion in a transition layer between positiveindex and negative-index media," Opt. Express 16, 18505-18513 (2008).

72. J. B. Pendry, D. Schurig, and D. R. Smith, "Controlling electromagnetic fields," Science 312, 1780-1782 (2006).

73. U. Leonhardt, "Optical conformal mapping," Science 312 , 1777-1780 (2006).

74. U. Leonhardt and T. G. Philbin, "General relativity in electrical engineering,” New J. Phys. 8, 247 (2006).

75. D. Schurig, J. J. Mock, B. J. Justice, S. A. Cummer, J. B. Pendry, A. F. Starr, and D. R. Smith, "Metamaterial electromagnetic cloak at microwave frequencies," Science 314, 977-980 (2006).

76. W. Cai, U. K. Chettiar, A. V. Kildishev, and V. M. Shalaev, "Optical cloaking with metamaterials," Nature Photon. 1, 224-227 (2007).

77. W. Cai, U. K. Chettiar, A. V. Kildishev, V. M. Shalaev, and G. Milton, "Nonmagnetic cloak with minimized scattering," Appl. Phys. Lett. 91, 111105 (2007).

78. M. Rahm, D. Schurig, D. A. Roberts, S. A. Cummer, D. R. Smith, and J. B. Pendry, "Design of electromagnetic cloaks and concentrators using form-invariant coordinate transformations of Maxwell's equations," Photonics
Nanostruct. Fundam. Appl. 6, 87-95 (2008).

79. A. V. Kildishev and V. M. Shalaev, "Engineering space for light via transformation optics," Opt. Lett. 33, 43-45 (2008).

80. W. X. Jiang, T. J. Cui, X. Y. Zhou, X. M. Yang, and Q. Cheng, "Arbitrary bending of electromagnetic waves using realizable inhomogeneous and anisotropic materials," Phys. Rev. E 78, 066607 (2008).

81. M. Rahm, D. A. Roberts, J. B. Pendry, and D. R. Smith, "Transformation-optical design of adaptive beam bends and beam expanders," Opt. Express 16, 11555-11567 (2008).

82. E. E. Narimanov and A. V. Kildishev, "Optical black hole: broadband omnidirectional light absorber," Appl. Phys. Lett. 95, 041106 (2009).

83. Y. Lai, J. Ng, H. Y. Chen, D. Han, J. Xiao, Z.-Q. Zhang, and C. T. Chan, "Illusion optics: the optical transformation of an object into another object," Phys. Rev. Lett. 102, 253902 (2009).

84. A. V. Kildishev, W. Cai, U. K. Chettiar, and V. M. Shalaev, "Transformation optics: approaching broadband electromagnetic cloaking," New J. Phys. 10, 115029 (2008).

85. L. S. Dolin, "On the possibility of comparison of threedimensional electromagnetic systems with nonuniform anisotropic filling," Izv. Vyssh. Uchebn. Zaved., Radiofiz. 4, 964-967 (1961).

86. A. J. Ward and J. B. Pendry, "Refraction and geometry in Maxwell's equations,” J. Mod. Opt. 43, 773-793 (1996).

87. J. Li and J. B. Pendry, "Hiding under the carpet: a new strategy for cloaking," Phys. Rev. Lett. 101, 203901 (2008).

88. J. Valentine, J. Li, T. Zentgraf, G. Bartal, and X. Zhang, "An optical cloak made of dielectrics," Nature Mater. 8, 568-571 (2009).

89. R. Liu, C. Ji, J. J. Mock, J. Y. Chin, T. J. Cui, and D. R. Smith, "Broadband ground-plane cloak," Science 323, 366-369 (2009).

90. L. H. Gabrielli, J. Cardenas, C. B. Poitras, and M. Lipson, "Silicon nanostructure cloak operating at optical frequencies," Nature Photon. 3, 461-463 (2009).

91. I. I. Smolyaninov, V. N. Smolyaninova, A. V. Kildishev, and V. M. Shalaev, "Anisotropic metamaterials emulated by tapered waveguides: application to optical cloaking," Phys. Rev. Lett. 102, 213901 (2009). 